\title{
Reduction of Tetrazolium Salt XTT with UHT-Treated Milk: Its Relationship with the Extent of Heat-Treatment and Storage Conditions
}

\author{
Hiroyuki Ukeda, ${ }^{1}$ Yukihiko Goto, ${ }^{1}$ Masayoshi Sawamura, ${ }^{1}$ Hirozo Kusunose, ${ }^{1}$ Hideaki $\mathrm{KAmIKADO}^{2}$ \\ and Toshiro KAMEI ${ }^{2}$ \\ ${ }^{1}$ Department of Bioresources Science, Faculty of Agriculture, Kochi University, Monobe B-200, Nankoku 783, Japan \\ ${ }^{2}$ Central Research Institute, Meiji Milk Products Co., Ltd., 1-21-3, Sakae-cho, Higashimurayama, Tokyo 189, Japan
}

Received May 17, 1995

\begin{abstract}
UHT-treated milk reduced $3^{\prime}-\{1-[$ (phenylamino)-carbonyl]-3,4-tetrazolium $\}$-bis(4-methoxy-6-nitro)benzenesulfonic acid hydrate (XTT). The reducibility was caused by the Maillard reaction intermediate between milk protein and lactose. First, the assay procedure of the XTT reduction was optimized using the heated model solution containing casein and lactose and UHT-treated milk. When the XTT reduction assay was applied to two kinds of UHT-treated milks under optimum conditions, the ability of each milk sample to reduce XTT significantly reflected the extent of the heat treatment as well as the evaluation method using the hydroxymethylfurfural (HMF) value. In contrast to the HMF value, the ability of UHT-treated milk gradually decreased depending upon the storage period and temperature. These results suggest not only that the present XTT assay is applicable to estimate the extent of heat treatment but also that the reducibility of the milk sample heat-treated under a given condition can serve to estimate the storage period if the storage temperature is known or vice versa. The method is much simpler (only mixing of the XTT solution containing menadione with milk sample) and quicker (about 20 min) with a satisfactory reproducibility than the conventional methods to estimate the extent of the Maillard reaction such as the lactulose or HMF determination.
\end{abstract}

Keywords: UHT-treated milk, Maillard reaction, tetrazolium salt

During the heat treatment and prolonged storage of foods, the reactions between amines and carbohydrates can lead to the formation of an undesirable flavor and color but also to a serious loss in nutritive value (Walstra \& Jenness, 1984; Finot et al., 1981). In milk, mainly lactose and the $\varepsilon$-amino groups of protein bound-lysine are involved in this Maillard reaction or non-enzymatic browning.

The change in milk during the Maillard reaction by the heating process can be estimated by a change in hydroxymethylfurfural (HMF) and lactulose concentration (Fink \& Kessler, 1988). As these concentrations increase with increasing heat treatment, they have been recognized to be the most promising indicators for the treatment (Keeney \& Bassette, 1959; Geier \& Klostermeyer, 1980). However, the determination methods are generally time-consuming and tedious.

A tetrazolium salt, nitroblue tetrazolium (NBT), is reduced at alkaline $\mathrm{pH}$ with Amadori product formed by the Maillard reaction between protein and reducing sugar (Johnson et al., 1982). The principle has been clinically applied to the determination of glycated protein such as serum albumin (Mashiba et al., 1992). However, the reduction occurred not only with the Amadori product but also with the browned derivatives of protein (Syrovy, 1994; Ghiggeri et al., 1988) and additionally the Amadori product is known to produce superoxide anion which can reduce NBT via the alkoxy radical at alkaline pH (Sakurai \& Tsuchiya, 1988). These facts have further complicated the interpretation of the NBT assay. The reduction of tetrazoliums generally occurs in milder conditions and for a shorter time compared with the determi- nation methods of HMF and lactulose. Therefore, the application of tetrazolium salts for the determination of the Maillard reaction product would be attractive if the assay could be carried out at neutral $\mathrm{pH}$ where such a secondary reaction does not normally occur.

The authors reported the microbial counting method of raw milk based on the reduction reaction of NBT mediated with menadione at neutral pH (Ukeda et al., 1995). We observed that, when the method was applied to commercially available UHT-treated milks, a similar reduction reaction occurred despite the fact that the milks contained no microorganisms. This result suggests that the Maillard reaction product in milk formed by UHT-treatment could reduce the tetrazolium salt at neutral $\mathrm{pH}$. In the present investigation, the reduction reaction of tetrazolium with UHT-treated milk has been applied to differentiate the extent of the thermal treatment of commercially available milk products.

Tetrazolium salts have proven useful as an indicator of reducing systems like the electron transport system (ETS) of microorganisms and also in a number of enzymatic assays (Altman, 1976). Bacteria with an active ETS reduce these redox dyes from a water soluble tetrazolium salt to a colored, water-insoluble formazan product. The formazan accumulates inside the cells, thus conveniently permitting its identification by microscopy. In an application of the tetrazolium salts to measure the reducing capacity of UHT-treated milk, however, the insolubility of formazan is unfavorable because the insolubility might lead to a heterogeneous dispersion of formazan in the milk. 
A new tetrazolium salt, sodium $3^{\prime}-\{1-[($ phenylamino $)$-carbonyl]-3,4-tetrazolium $\}$-bis(4-methoxy-6-nitro)benzenesulfonic acid hydrate (XTT), with a corresponding watersoluble formazan has been synthesized (Paull et al., 1988). This tetrazolium salt appeared promising as viability indicator in assays of bacteria (Roslev \& King, 1993) and eucaryotic cells (Tellier et al., 1992).

Here we apply the XTT to evaluate the extent of heating treatment of UHT-treated milk. Interestingly, the reducing ability of packaged UHT-treated milk during storage significantly decreased with increasing storage time and temperature, suggesting that the present XTT assay could also be used to estimate the storage period of the UHT-treated milk if the storage temperature is known or vice versa.

\section{Materials and Methods}

Reagents NBT, menadione (2-methyl-1,4-naphthoquinone) and HMF were obtained from Wako Pure Chemical Industries (Osaka, Japan). XTT was purchased from Polyscience (Warrington, PA). Lactose monohydrate and nutrose (casein sodium) were obtained from Nacalai Tesque (Kyoto, Japan). All other reagents were of the highest grade commercially available. Milli-Q water was used in all procedures.

Milk samples Two kinds of UHT-treated milks with a fat content of $3.5 \%$ and 3.6\%, supplied by Meiji Milk Products Co., were selected as the test products for all investigations. The UHT treatment of the samples was carried out at $130^{\circ} \mathrm{C}$ for $2 \mathrm{~s}$ (Milk A) and at $140^{\circ} \mathrm{C}$ for $3 \mathrm{~s}$ (Milk B) by an indirect heat exchange apparatus containing steam heated and water cooled capillary tubes. Milk A and B were produced from the identical milk source. Milk B was manufactured as LL milk. The package size of the milk samples was $200 \mathrm{ml}$ (Milk A) and $250 \mathrm{ml}$ (Milk B) and each sample was drawn just after opening the package. The packaged milk was stored in the dark at a given temperature. In one experiment, a commercially available pasteurized milk was used. The heat treatment of the product was carried out at $65^{\circ} \mathrm{C}$ for $30 \mathrm{~min}$ (Milk C).

Model solution A given amount of lactose and casein was dissolved in water followed by heating in a water bath or autoclaving for $20 \mathrm{~min}$ at $120^{\circ} \mathrm{C}$. The reaction conditions of XTT were optimized using the autoclaved model solution containing lactose $(4.6 \mathrm{w} / \mathrm{v} \%)$ and casein $(2.6 \mathrm{w} / \mathrm{v} \%)$. The $\mathrm{pH}$ of the autoclaved model solution was 6.26. A new batch of this solution was prepared for each experiment and stored at $5^{\circ} \mathrm{C}$ for no longer than $24 \mathrm{~h}$.

Assay procedures XTT was dissolved in $0.2 \mathrm{M}$ potassium phosphate buffer saturated with menadione (ca. 0.55 $\mathrm{mm})$. Samples $(1.0 \mathrm{ml})$ were pipetted into a $4.2-\mathrm{cm}$ diameter glass petri dish and the XTT solution $(1.0 \mathrm{ml})$ was added into it. The color of each mixture was determined using a color reflectance meter CR-200 (Minolta, Tokyo, Japan) and the values $L, a$ and $b$ of the CIELAB color difference equation developed from the Hunter color measurement system were recorded. The color change of each sample was expressed as a difference in $L, a$ and $b$ values between 0 and 20 min after the addition of the XTT solution unless otherwise indicated. The change in the $a$ value was chosen as an index of the XTT color change, as the change was bigger compared with that of the $L$ and $b$ values. A white calibration plate furnished by the manufacturer of the apparatus was used as a calibration standard. In one experiment, NBT was used instead of XTT. Except for the fact that NBT was dissolved in $0.2 \mathrm{M}$ potassium phosphate buffer $(\mathrm{pH} 7.0)$ at a concentration of $1 \mathrm{~mm}$, the procedure was same as that of XTT.

Microbial count of milk The number of microorganisms in a milk sample was measured by plating suitably diluted portions of a milk and counting colonies that appeared after $48 \mathrm{~h}$ of incubation of $30^{\circ} \mathrm{C}$.

Determination of HMF HMF was colorimetrically determined according to the method described by Keeney and Bassete (1959). In this method, bound HMF was released by heating with dilute oxalic acid. After the precipitation of the protein with trichloroacetic acid and filtering, the yellow coloring formed after the addition of thiobarbituric acid was spectrophotometrically measured at $443 \mathrm{~nm}$. Determinations were carried out in duplicate.

\section{Results and Discussion}

We previously reported that raw milk samples containing no microorganisms showed no color change during the reaction with NBT under the previously described conditions (Ukeda et al., 1995). However, the UHT-treated milk sample (Milks A and B) showed a certain color change by the reaction with NBT, although it contained no microorganisms. The extent of the color change was equivalent to a microbial count of $10^{5} \mathrm{cfu} / \mathrm{ml}$ or more if it was a raw milk sample (Ukeda et al., 1995). When the milk sample was mixed with XTT solution, an intense orange color showing the reduction of XTT appeared in the reaction mixture as well as during the reaction with NBT. The extent of the color change was clearly stronger than that of NBT. This result suggests that the thermal treatment of milk causes the formation of a substance that can reduce XTT. A similar reducibility of XTT was observed in the heated model solution containing both casein and lactose, while the reduction of XTT did not occur when they were separately heated (Fig. 1). It was suggested, therefore, that the Maillard reaction between sugar and protein in milk during heating was responsible for the reduction of XTT. As XTT indicated a more intense color change for both a thermal-treated milk and the model solution in comparison with NBT, XTT was used in a subsequent experiment to examine the applicability of the reaction to estimate the extent of the heating process of milk.

Effect of reaction conditions of XTT with the model solution In order to exactly know the difference in the reducibility of XTT between the thermal-treated and nontreated coexistence system of protein and sugar, the model solution containing casein and lactose was used in the optimization experiments. Figure 2 depicts the effect of XTT concentration on the change in the $a$ value. A rapid increase in the change in the $a$ value was observed in the concentration up to $0.5 \mathrm{~mm}$ and a tendency to level off was recognized above this concentration. The $\mathrm{pH}$ dependence is shown in Fig. 3. Although the change in the $a$ value increased at higher $\mathrm{pH}$, the heated model solution showed a significantly larger change in the $a$ value than that of the non-treated one even at neutral $\mathrm{pH}$. As a general property, the tetrazolium salt can be 


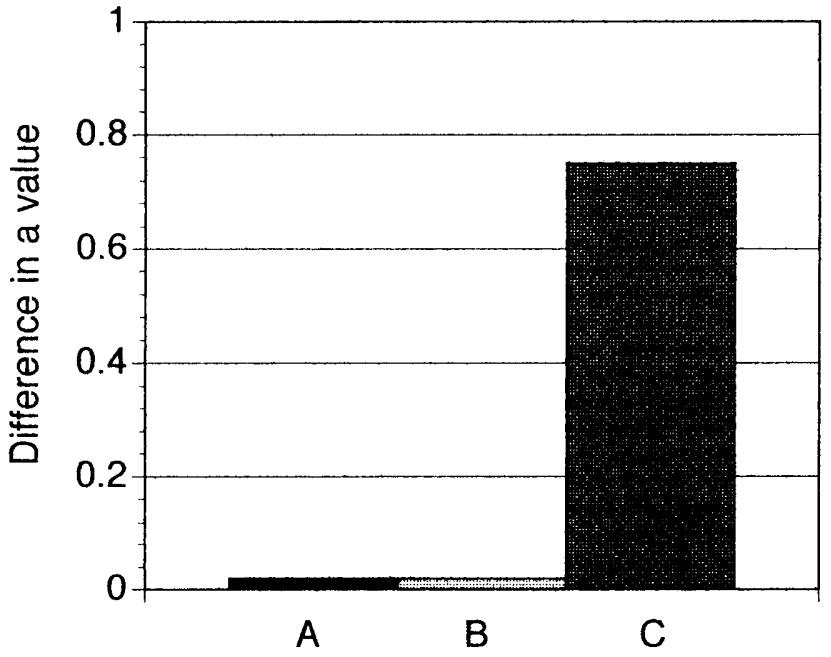

Fig. 1. Comparison of the ability of the model solution containing casein and/or lactose to reduce XTT. The composition of the model solution was as follows; A, $4.6 \%$ lactose; B, $2.6 \%$ casein; C, $4.6 \%$ lactose and $2.6 \%$ casein.

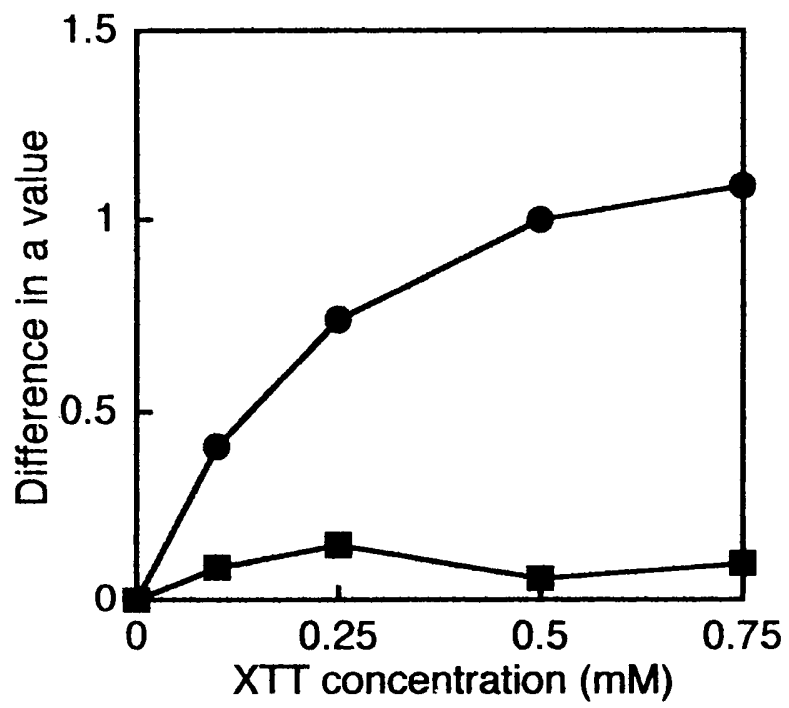

Fig. 2. Effect of the XTT concentration on the difference in $a$ value. XTT was dissolved in $0.2 \mathrm{M}$ potassium phosphate buffer $(\mathrm{pH} 7.0$ ) saturated with menadione. The heat-treated $(\bullet)$ model solution was compared with nontreated one $(\mathbf{\square})$.

reduced by reducing sugars such as lactose in alkaline $\mathrm{pH}$ (Altman, 1976). In addition, the Maillard reaction product changes to a reactive species like an alkoxy radical at alkaline $\mathrm{pH}$ (Sakurai \& Tsuchiya, 1988). Therefore, the use of alkaline $\mathrm{pH}$ could be unfavorable for monitoring the interaction between casein and lactose when heating. Figure 4 shows the effect of the concentration of menadione on the change in the $a$ value. The addition of menadione at the saturation level raised the change in the $a$ value to 1.5 -fold. Menadione has been known as an effective substrate of ETS of microorganisms and applied to the determination of ETS activity using a combination of tetrazolium salts (Ukeda et al., 1995; Roslev \& King, 1993). We observed that the addition of menadione at the saturation level increased the reaction rate to about two-fold (Ukeda et al., 1995). Also, its effectiveness has been shown as a redox mediator between dihydropyridine

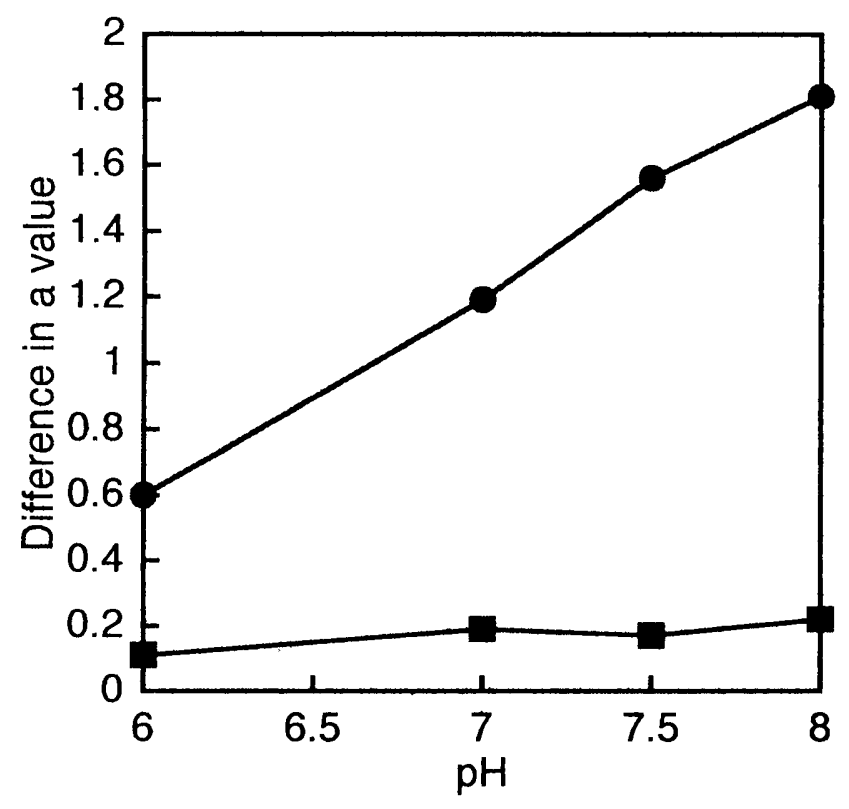

Fig. 3. Effect of $\mathrm{pH}$ of the buffer in which XTT was dissolved on the difference in $a$ value. The XTT concentration was $0.5 \mathrm{~mm}$. The heat-treated (-) model solution was compared to the non-treated one( $\boldsymbol{\square})$. In this experiment, 0.2 $\mathrm{M}$ potassium phosphate buffer was used at each $\mathrm{pH}$. The $\mathrm{pH}$ after the addition of the model solution was confirmed to be the same as that of each buffer.

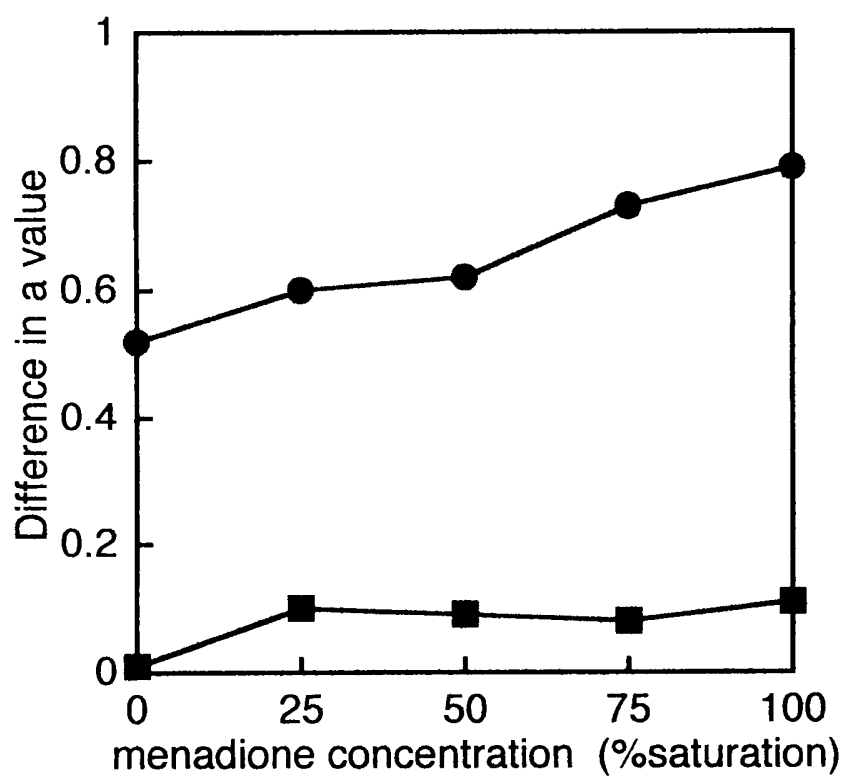

Fig. 4. Effect of the concentration of menadione on the difference in $a$ value. The XTT was dissolved in $0.2 \mathrm{M}$ potassium phosphate buffer $(\mathrm{pH} \mathrm{7.0)}$ at the concentration of $0.5 \mathrm{~mm}$. The heat-treated $(\bullet)$ model solution was compared to the non-treated one (

and molecular oxygen in the determination of protein based on the use of glutaraldehyde (Ukeda et al., 1986). Judging from the result that the effect of the addition of menadione on the change in the $a$ value as shown in Fig. 4 was less than that on the ETS of microorganisms, it seems likely that menadione partly plays the role as a redox mediator between the product formed by the Maillard reaction during heating and XTT. The effect of the reaction temperature on the change in the $a$ value was examined in the range of $20-37^{\circ} \mathrm{C}$ (Fig. 5). There was no significant difference in the change in the $a$ value between 25 and $37^{\circ} \mathrm{C}$. 


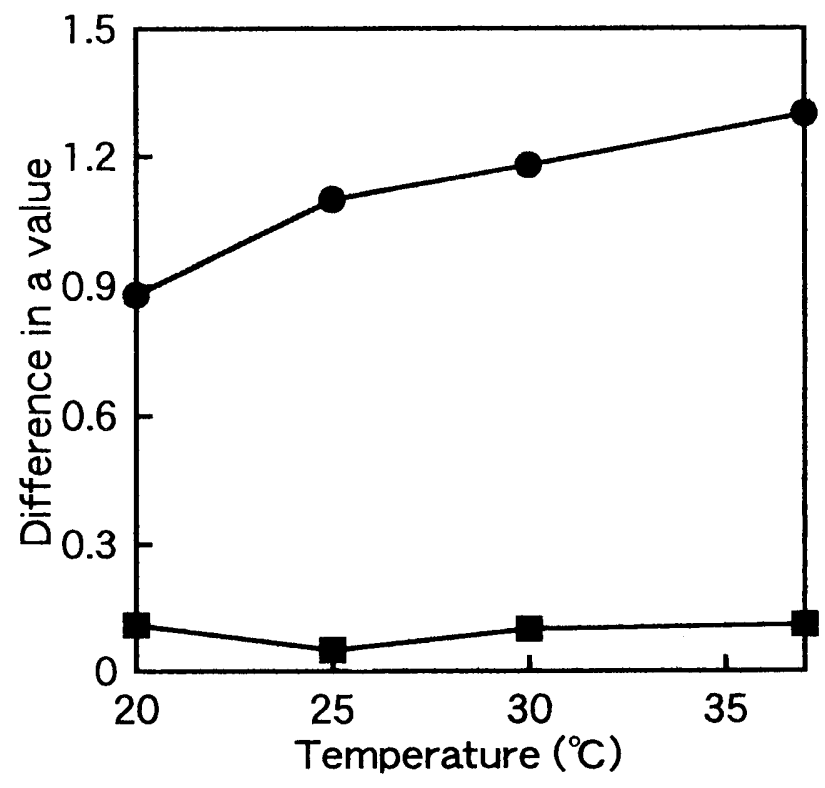

Fig. 5. Effect of the reaction temperature on the difference in $a$ value. The XTT $(0.5 \mathrm{~mm})$ was dissolved in $0.2 \mathrm{M}$ potassium phosphate buffer $(\mathrm{pH} 7.0)$ saturated with menadione. The heat-treated $(\bullet)$ model solution was compared to the non-treated one ( $\boldsymbol{\square})$.

From these results, the following reaction conditions were chosen: XTT concentration, $0.5 \mathrm{~mm}$; reaction $\mathrm{pH}, 7.0$; menadione concentration, saturation level (approximately $0.55 \mathrm{~mm}$ ); and reaction temperature, $25^{\circ} \mathrm{C}$.

Relationship between the heating conditions and the $X T T$ reducibility When the concentration of lactose was changed in the range of 0 to $6.9 \%$, the change in the $a$ value linearly increased with the increase in the concentration (Fig. 6). The dependence of the concentration of casein on the change in the $a$ value was similar to that of the lactose concentration (Fig. 7). When the model solutions containing lactose and casein were heated at 80 or $90^{\circ} \mathrm{C}$, the change in the $a$ value increased with an increase in the heating time and temperature. The Maillard reaction is promoted by the heating time and temperature and an increase in the solid concentration (Walstra \& Jenness, 1984). It was suggested, therefore, that the change in the $a$ value during the reaction with XTT could reflect the extent of the Maillard reaction in the heated model solution.

Figure 8 depicts the dependence of $\mathrm{pH}$ of the model solution upon heating on the change in the $a$ value. The maximum change in the $a$ value was recognized in the range of $\mathrm{pH} 7.5$ to 8 . It was noted, on the other hand, that the extent of browning linearly increased with increasing $\mathrm{pH}$ of the model solution (Fig. 8). This result means that the substance which reduces XTT is not the end products of the browning reaction such as melanoidin but the intermediate formed during the reaction.

Effect of the reaction conditions of XTT with heated milk samples The optimum conditions chosen by using the model solution may not necessarily be the same as those of heated milk because the components other than casein and lactose might be involved in the reduction of XTT. A similar experiment was conducted using the UHT-treated milk sample (Milk B).

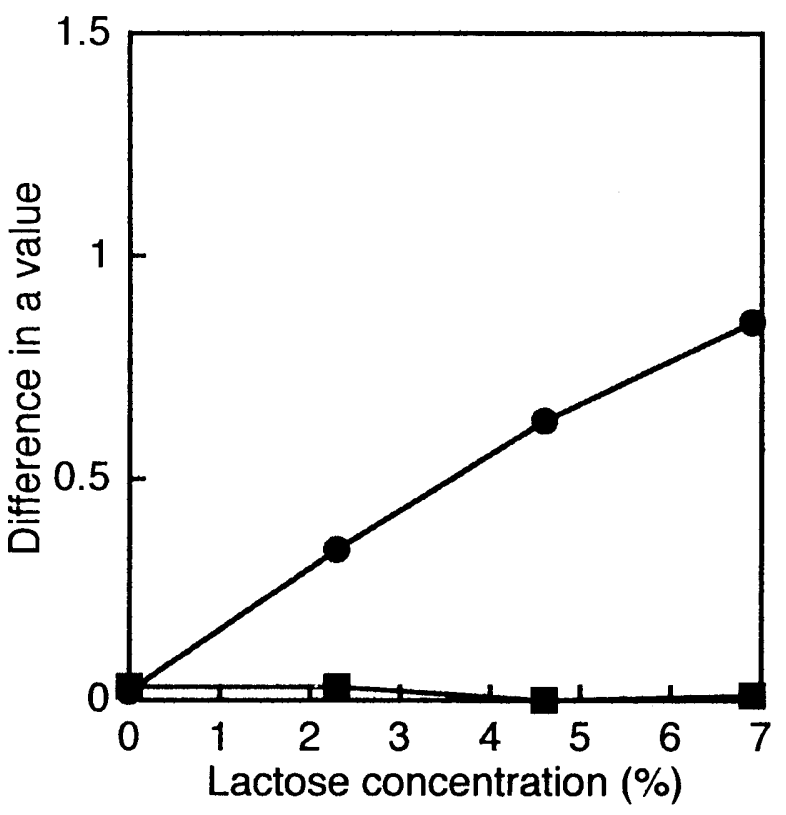

Fig. 6. Dependence of the lactose concentration in the model solution on the difference in $a$ value. The concentration of casein was fixed at $2.6 \%$. The heat-treated ( $)$ model solution was compared to the non-treated one ( $\boldsymbol{\square})$.

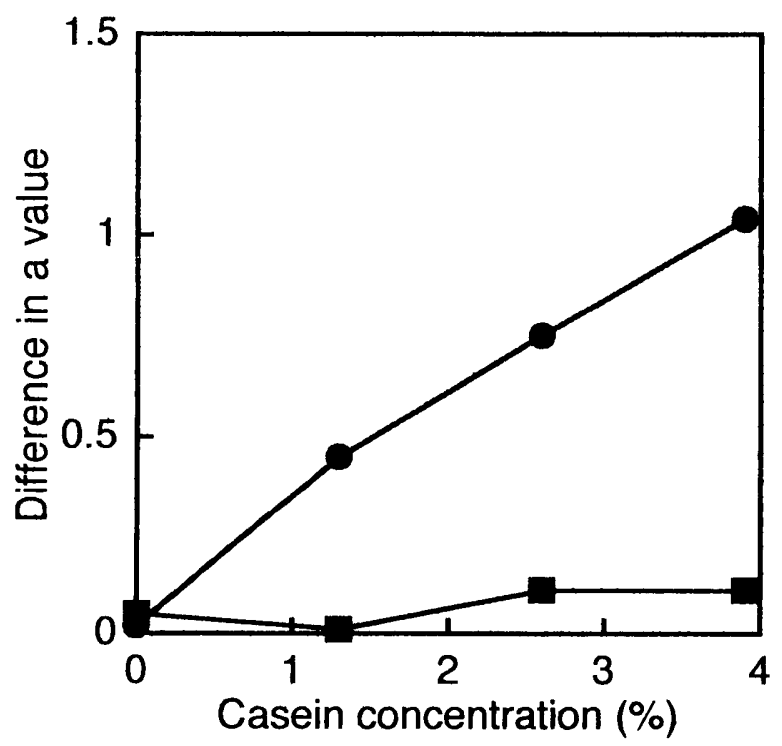

Fig. 7. Dependence of the casein concentration in the model solution on the difference in $a$ value. The concentration of lactose was fixed at $4.6 \%$. The heat-treated (๑) model solution was compared to the non-treated one (ם)

The effects of the concentration of XTT and menadione were almost the same as those for the model solution. Figure 9 indicates the dependence of the reaction $\mathrm{pH}$ on the change in the $a$ value. The change increased with increasing $\mathrm{pH}$ as well as the results shown in Fig. 3, but the tendency to increase at a $\mathrm{pH}$ higher than 7 was smaller than that using the model solution. From these results, the conditions chosen in the reaction of XTT with the model solution were also selected as an optimum in the reaction with UHT-treated milk. Furthermore, these results suggest that the property of the substance responsible for the reduction of XTT in a heated milk is similar to that of the reaction products formed from casein and lactose during the thermal treatment of the model solution.

Relationship between the extent of the thermal treat- 


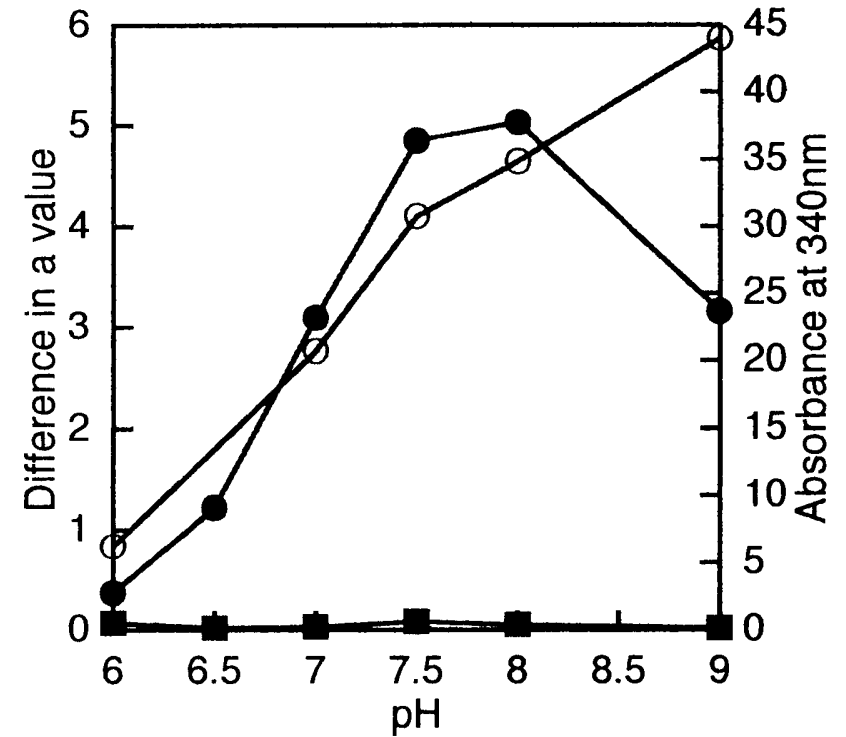

Fig. 8. Dependence of $\mathrm{pH}$ of the model solution upon heating on the difference in $a$ value and the browning. The model solution contained of $4.6 \%$ lactose and $2.6 \%$ casein. The difference in $a$ value of the heat-treated $(\bullet)$ model solution was compared to that of the non-treated one (1). The extent of browning of the heat-treated model solution was monitored as the absorbance at $340 \mathrm{~nm}(\mathrm{c})$.

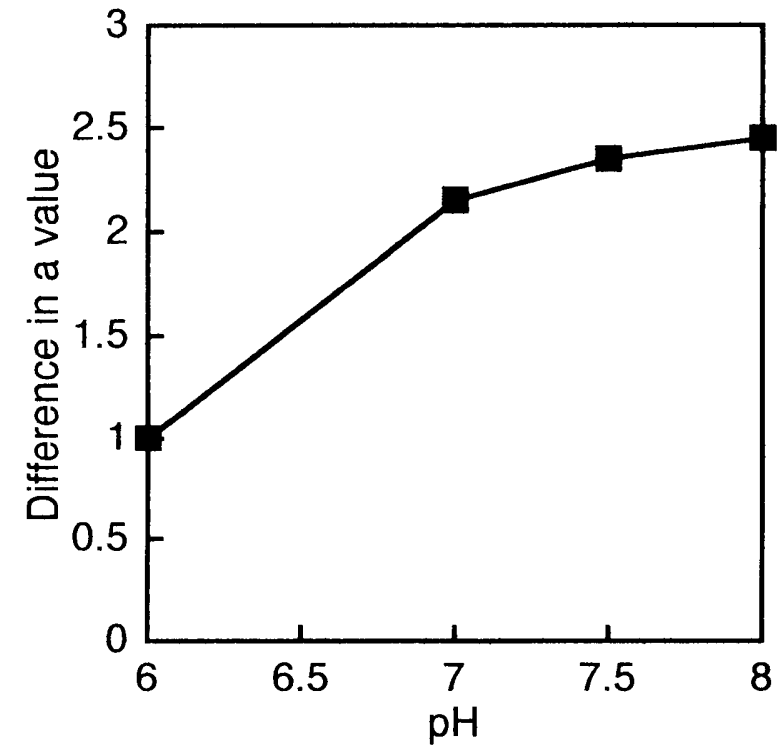

Fig. 9. $\mathrm{pH}$ dependence of the ability of the UHT-treated milk to reduce XTT. XTT was dissolved in $0.2 \mathrm{M}$ potassium phosphate buffer with different $\mathrm{pHs}$.

ment and the reduction of $X T T$ The reaction products formed as a consequence of the Maillard reaction have been confirmed by the determination of the HMF value (Fink \& Kessler, 1988). The determination method involves the conversion of early intermediates of the browning reaction such as 1-amino-1-deoxy-fructose to HMF by the heating of the milk with added oxalic acid. Kessler and Fink (1986) investigated the relationship between the HMF value and heating process over a wide range of times and temperatures. According to the results, the HMF value clearly increases with the severity of the heating treatment.

The HMF values of Milks A and B were 8.95 and $12.6 \mu \mathrm{M}$, respectively. As was expected, Milk B showed a higher value. Figure 10 indicates the change in the $a$ value of each milk

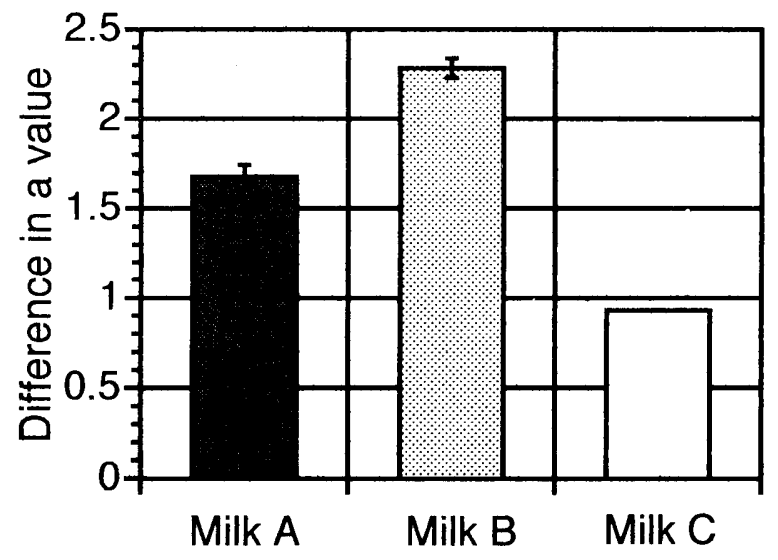

Fig. 10. Comparison of the ability of the UHT-treated milks (Milk A and B) and pasteurized milk (Milk C) to reduce XTT. Each bar for the UHT-treated milks represents the mean \pm SD of four different lots.

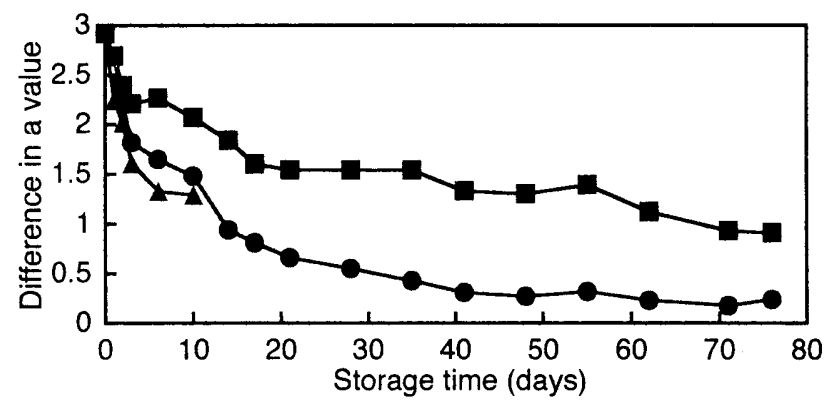

Fig. 11. Relationship between the difference in $a$ value of the UHT-treated milk (Milk B) and the storage time and temperature. The UHT treated milk was stored at $5^{\circ} \mathrm{C}(\boldsymbol{\square}), 20^{\circ} \mathrm{C}(\bullet)$, or $30^{\circ} \mathrm{C}(\mathbf{\Delta})$

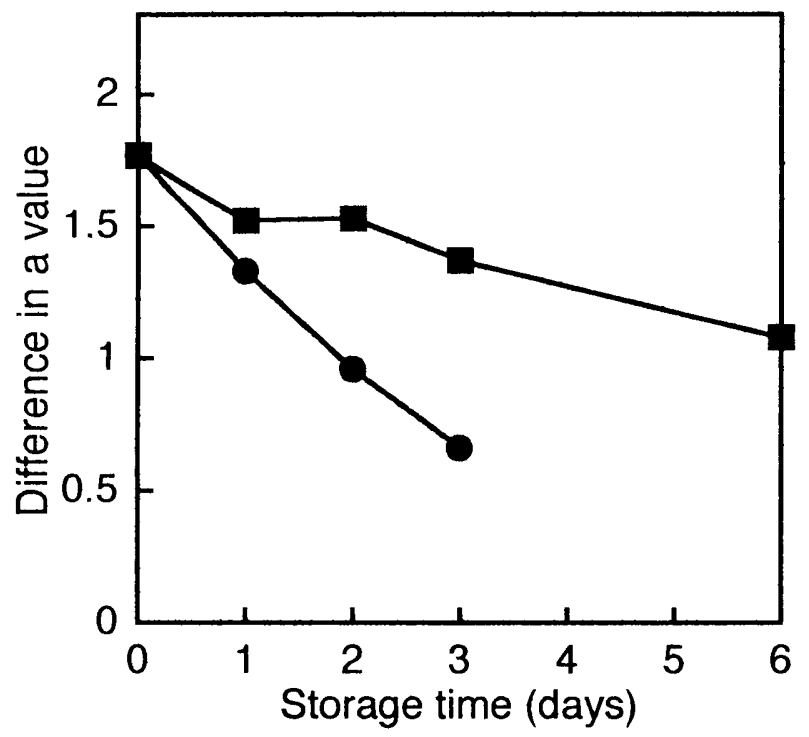

Fig. 12. Relationship between the difference in a value of the UHT-treated milk (Milk A) and the storage time and temperature. The UHT treated milk was stored at $5^{\circ} \mathrm{C}(\boldsymbol{a})$ or $20^{\circ} \mathrm{C}(\bullet)$

sample when the present XTT reduction assay was applied to Milks A and B. The value was indicated as the average of four different lots and each determination was carried out on the first day after UHT-treatment. The statistical analysis by Student's t test showed that there was a significant difference between them at the $0.05 \%$ level. This result suggests that the present XTT reduction assay makes it possible to assess the amount of thermal stress that a particular heat treatment 
delivers to the milk as well as the HMF value. The relative standard deviation of the present XTT assay was $3.9 \%(n=9)$.

Similarly, the XTT reduction assay was applied to a pasteurized milk (Milk C). As shown in Fig. 10, the pasteurized milk sample also showed the reducibility of XTT although the change in the $a$ value was much smaller than that of the UHT-treated milk (Milks A and B). This result suggests that the present XTT assay can detect the quality change in milk induced by the conditions of pasteurization as well as by the UHT treatment.

Effect of storage of UHT-treated milk on the reduction of XTT The HMF value of Milk B did not significantly change during its storage for 60 days at $5^{\circ} \mathrm{C}$, being consistent with the result reported by Fink and Kessler (1988). Lactulose concentration (Geier \& Klostermeyer, 1980), another acceptable indicator for the analytical control of heat treatment, was also known to be constant throughout storage periods of at least 70 days (Fink \& Kessler, 1988). In contrast, the $a$ value of Milk B by the XTT assay decreased with storage time (Fig. 11). The rate of the decrease in the $a$ value was clearly higher at higher temperatures. The half-life periods of the XTT reducibility of Milk B during storage at 5,20 and $30^{\circ} \mathrm{C}$ were 40,15 and 3 days, respectively. Another set of experiments using Milk B manufactured as another lot gave the same results. Similarly, the XTT reducibility of Milk A showed the tendency to gradually decrease depending upon the storage temperature (Fig. 12). These results strongly suggest that, when the present XTT assay is applied to the milk sample heat-treated under a given condition, the result can serve to estimate the storage period after heat treatment if the storage temperature is known or vice versa. At the same time, these results mean that a substance which reduces XTT in UHT-treated milk is never identical to the browning intermediates detected as the HMF value. The elucidation of the substance is currently under study.

\section{Conclusions}

Interpretation of the results for any loss of a constituent requires the measurement of the initial content of that component. Therefore, it seems better to use reaction products newly formed during heating as an indicator for the intensity of heat treatment. The products should be formed in sufficient amounts to clearly differentiate between the various heat treated milks. The XTT reducibility satisfactorily meets these demands as well as the HMF value and lactulose concentration.

The present XTT assay is much easier and quicker compared with the determinations of $\mathrm{HMF}$ value and lactulose concentration. No requirement of a special apparatus for heating and incubating and of a special or heatsensitive reagent such as enzymes would permit the use of the present method outside the laboratory. As XTT is a relatively expensive reagent, the development of a modified method using a micro titre plate is in progress in order to diminish the total assay volume. Using the modified method, a more detailed investigation will be done about the relationship between the XTT reducibility and heating process over a wide range of times and temperatures.

Another attractive property of the present XTT assay is based on the observation that the reducibility of UHT-treated milk decreased during storage. A difference in the $a$ value between just after UHT-treatment and after storage should reflect the storage period and temperature of the product. Hence, the present XTT assay can serve as a feasible method to estimate the storage conditions of UHT-treated milk.

\section{References}

Altman, F.P. (1976). Tetrazolium salts and formazans: A review. Prog. Histochem. Cytochem., 9, 1-56.

Fink, R. and Kessler, G. (1988). Comparison of methods for distinguishing UHT treatment and sterilization of milk. Milchwissenschaft, 43, 275-280

Finot, P.A., Deutsch, R. and Bujard, E. (1981). The extent of the Maillard reaction during the processing of milk. Prog. Food Nutr. Sci. 5, 345-355.

Geier, H. and Klostermeyer, H. (1980). Enzymatische Bestimmung von Lactulose. Z. Lebensm. Unters. Forsch., 171, 443-445.

Ghiggeri, G.M., Candiano, G., Ginevri, F., Oleggini, R., Piccardo, M.T., Bertelli, R., Perfumo, F. and Gusumano, R. (1988). Spectrophotometric determination of browning products of glycation of protein amino groups based on their reactivity with nitro blue tetrazolium salts. Analyst, 113, 1101-1104.

Johnson, R.N., Metcalf, P.A. and Baker, J.R. (1982). Fructosamine: a new approach to the estimation of serum glycosylprotein. An index of diabetic control. Clin. Chim. Acta, 127, 87-95.

Keeney, M. and Bassette, R. (1959). Detection of intermediate compounds in the early stages of browning reaction in milk products. J. Dairy Sci., 42, 945-960.

Kessler, H.-G. and Fink, R. (1986). Changes in heated and stored milk with an interpretation by reaction kinetics. J. Food Sci., 51, 1105-1111.

Kumetat, K., Beeby, R. and Morris, A.K. (1956). A note on the reducing groups of "Instant" milk powders. Aust. J. Dairy Technol., 11, 113-115.

Mashiba, S., Uchida, K., Okuda, S. and Tomita, S. (1992). Measurement of glycated albumin by the nitroblue tetrazolium colorimetric method. Clin. Chim. Acta, 212, 3-15.

Paull, K.D., Shoemaker, R.H., Boyd, M.R., Parsons, J.L., Risbood, P.A., Barbera, W.A., Sharma, M.N., Baker, D.C., H and, E., Scudiero, D.A., Monks, A., Alley, M.C. and Grote, M. (1988). The synthesis of XTT: a new tetrazolium reagent that is bioreducible to a water-soluble formazan. J. Heterocycl. Chem., 25, 911-913.

Roslev, P. and King, G.M. (1993). Application of a tetrazolium salt with a water-soluble formazan as an indicator of viability in respiring bacteria. Appl. Environ. Microbiol., 59, 2891-2896.

Sakurai, T. and Tsuchiya, S. (1988). Superoxide production from nonenzymatically glycated protein. FEBS Lett., 236, 406-410.

Syrovy, I. (1994). Glycation of albumin: reaction with glucose, fructose, galactose, ribose or glyceraldehyde measured using four methods. J. Biochem. Biophys. Methods, 28, 115-121.

Tellier, R., Krajden, M., Grigoriew, G.A. and Campbell, I. (1992) Innovative endpoint determination system for antifungal susceptibility testing of yeasts. Antimicrob. Agents Chemother., 36, 1619 1625

Ukeda, H., Miyazaki, E., Matsumoto, K. and Osajima, Y. (1986). Application of glutaraldehyde to amperometric determination of protein in dairy products. Anal. Chem., 58, 2975-2978.

Ukeda, H., Goto, Y., Sawamura, M., Kusunose, H. and Kamei, T. (1995). Estimation of microbial count in raw milk based on a colorimetric reaction with vitamin $\mathrm{K}_{3}$ and tetrazolium. Nippon Shokuhin Kagaku Kogaku Kaishi, 42, 627-633 (in Japanese).

Walstra, P. and Jenness, R. (1984). Heating. In "Dairy Chemistry and Physics." John Wiley \& Sons, New York, pp. 162-185. 